\title{
Measuring Probabilistic Reaction Norms for Age and Size at Maturation
}

Mikko Heino (mikko@imr.no)

Ulf Dieckmann (dieckman@iiasa.ac.at)

Olav Rune Godø (olav.rune.godoe@imr.no)

\section{Approved by}

Arne Jernelöv (jernelov@iiasa.ac.at)

Acting Director, IIASA

April 2002 Institute, its National Member Organizations, or other organizations supporting the work. 


\section{Contents}

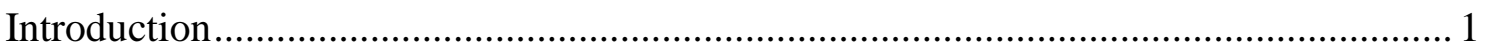

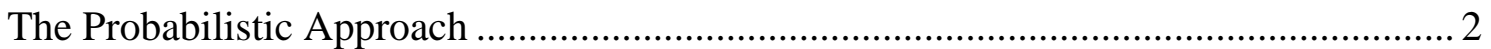

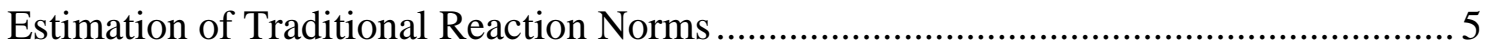

Estimation of Probabilistic Reaction Norms …........................................................... 7

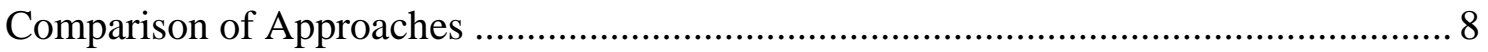

Example: Reaction Norms of Northeast Arctic Cod …................................................. 9

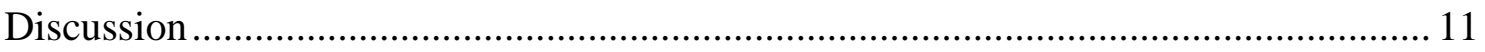

Appendix: Logistic-Regression Models for Maturation Reaction Norm ....................... 16

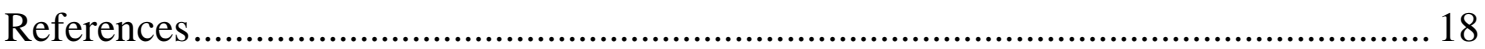




\begin{abstract}
We present a new probabilistic concept of reaction norms for age and size at maturation that is applicable when observations are carried out at discrete time intervals. This approach can also be used to estimate reaction norms for age and size at metamorphosis or at other ontogenetic transitions. Such estimations are critical for understanding phenotypic plasticity and life-history changes in variable environments, for assessing genetic changes in the presence of phenotypic plasticity, and for calibrating size- and age-structured population models. We show that previous approaches to this problem, based on regressing size against age at maturation, give results that are systematically biased when compared to the probabilistic reaction norms. The bias can be substantial and is likely to lead to qualitatively incorrect conclusions; it is caused by failing to account for the probabilistic nature of the maturation process. We explain why, instead, robust estimations of maturation reaction norms ought to be based on logistic regression, or on other statistical models that treat the probability of maturing as a dependent variable. We demonstrate the utility of our approach with two examples. First, the analysis of data generated for a known reaction norm highlights some crucial limitations of previous approaches. Second, application to the Northeast Arctic cod (Gadus morhua) illustrates how our approach can be used to shed new light on existing real-world data.
\end{abstract}




\title{
About the Authors
}

\author{
Mikko Heino \\ Institute of Marine Research \\ Box 1870 Nordnes \\ N-5817 Bergen, Norway \\ and
}

Adaptive Dynamics Network

International Institute for Applied Systems

A-2361 Laxenburg, Austria

and

University of Helsinki

Department of Ecology and Systematics

FIN-00014 Helsinki, Finland

\author{
Ulf Dieckmann \\ Adaptive Dynamics Network \\ A-2361 Laxenburg, Austria \\ Olav Rune God $\varnothing$ \\ Institute of Marine Research \\ Box 1870 Nordnes \\ N-5817 Bergen, Norway
}

International Institute for Applied Systems Analysis

\section{Acknowledgments}

We thank T. Kawecki for comments that led to substantial refinements of this paper. We also thank R. Law for inspiring discussion and S. Barot, W. Blanckenhorn, D. Claessen, B. Ernande, and T. Van Dooren for valuable advice and comments on the earlier versions of the paper. Mikko Heino and Ulf Dieckmann wish to thank the Institute of Marine Research (Norway) for an opportunity to participate in a research cruise on ' $R / \mathrm{V}$ Johan Hjort', during which the initial draft of this paper was written. Mikko Heino acknowledges financial support by the Academy of Finland (Project 45928). Collaboration on this study has been fostered by the European Research Training Network ModLife (Modern Life-History Theory and its Application to the Management of Natural Resources), supported by the Fifth Framework Programme of the European Community (Contract Number HPRN-CT-2000-00051). 


\title{
Probabilistic Maturation Reaction Norms for Age and Size at Maturation
}

\author{
Mikko Heino \\ Ulf Dieckmann \\ Olav Rune Godø
}

\section{Introduction}

Age and size at maturation are key life-history traits and affect growth rate, fecundity, and survival later in life (Roff 1992; Stearns 1992). However, individuals within a population typically differ in their growth patterns, and maturation occurs at different ages and sizes for individuals that follow different growth trajectories. The covariation of age and size at maturation is often described by plotting the critical size at which maturation occurs as a function of age (Fig. 1A). It is customary to refer to such relationships as the reaction norm for age and size at maturation (Stearns and Koella 1986). In this paper we adhere to the convention of using the term reaction norm for this population-level relation, which is justified if variability in growth trajectories is mainly due to environmental effects (see Discussion).

The concept of the reaction norms for age and size at maturation was originally developed by Stearns and co-workers (Stearns and Crandall 1984; Stearns and Koella 1986) as a theoretical tool to describe how individuals deal with environmental variability in growth rates. In essence, the concept was based on models that assume that maturation is a deterministic process - that is, the onset of sexual maturity is fully determined by age and size of an individual. In the real world, however, maturation invariably involves stochastic elements (Bernardo 1993).

It is important to notice that the probabilistic nature of maturation has never received careful consideration in the context of maturation reaction norms. However, the proper treatment of variability in maturation becomes critical when maturation reactions norms are estimated from real data. In this paper we extend the concept of maturation reaction norms in a way that involves stochasticity of maturation as an explicit part of the reaction norm description. We restrict ourselves to examples in which observations are made periodically. Such periodicity may arise naturally (i.e., due to seasonality, or to tidal and diurnal cycles), or it may be caused by the practical constraints of the experimenter. However, in the absence of natural periodicity, an approach in which the reaction norm is expressed as age- and size-dependent maturation rates may be preferable. This complementary approach unfortunately lies beyond the scope of this paper.

For the sake of brevity we focus here on the process of maturation; similar considerations apply to reaction norms for other ontogenetic transitions. We emphasize two main points. First, the probabilistic refinement of the maturation reaction norm is a 
helpful tool for coping with variability in empirical data. Second, estimation of the probabilistic reaction norm requires information on both immature and maturing individuals, in contrast to the traditional methods.

This paper is structured as follows. We start by introducing the probabilistic concept for the reaction norm for age and size at maturation; the new concept includes the traditional deterministic concept as a special case. We continue by showing that the relationship between age and size at maturation is often remarkably uninformative with respect to the probabilistic reaction norm for age and size at maturation. The next section describes how estimates of the probabilistic maturation reaction norm can be obtained by logistic regression, requiring data on immature as well as maturing individuals. Analysis of data derived from known reaction norms then reveals the crucial differences between our approach and previous concepts of maturation reaction norms. In the penultimate section we estimate probabilistic maturation reaction norms for the Northeast Arctic cod (Gadus morhua L.), one of the economically most important fish stocks in the world.

\section{The Probabilistic Approach}

We consider data collected by sampling a cohort of individuals at regular intervals. In each of the repeated samples, three classes of individuals are distinguished: immature individuals, maturing individuals (or, equivalently, newly matured individuals), and mature individuals that have matured during an earlier time interval. For the sake of brevity, we use the term 'maturing' to refer to all individuals that would have been classified as immature at the previous time of observation and that have reached maturation by the current time of observation.

In a deterministic world, maturation would occur with certainty once growth trajectories reach the reaction norm for age and size at maturation (Fig. 1A). However, maturation is a complex process that is also influenced by factors other than age and size, such as current resource availability and body reserves (Bernardo 1993). Because of such extra factors, there will be some variability in the size of maturing individuals at a given age (Fig. 1B). As long as the cohort is not yet fully mature, there are immature individuals that, in principle, could also have matured during the considered time interval. The fact that some individuals in a certain age and size class may mature while others do not, highlights the probabilistic nature of the maturation process. The age and size dependence in the propensity to mature can be described by age- and sizedependent probabilities of maturation. We take the probability of an immature individual to mature within a considered age and size class as the basis for a probabilistic definition of the reaction norm for age and size at maturation.

It is important to notice that the probability of maturing depends on the time interval between the observations: the shorter the length of these intervals, the lower is the corresponding maturation probability. This is unproblematic if natural periodicity, for example through the effects of seasonality, constrains maturation events to occur at certain times, and the observations are carried out by respecting this periodicity. However, in the absence of natural periodicity, one should highlight the dependence of maturation probabilities on the time interval chosen between consecutive observations. 

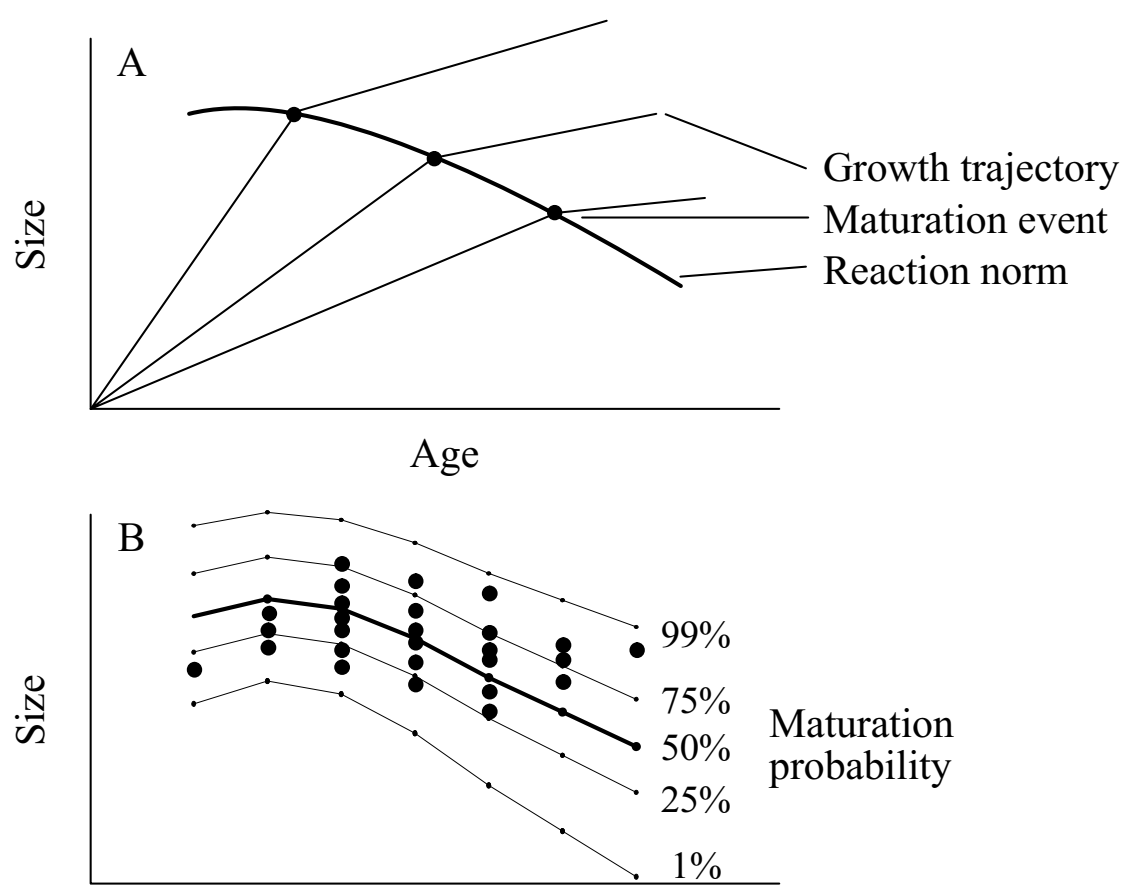

Age

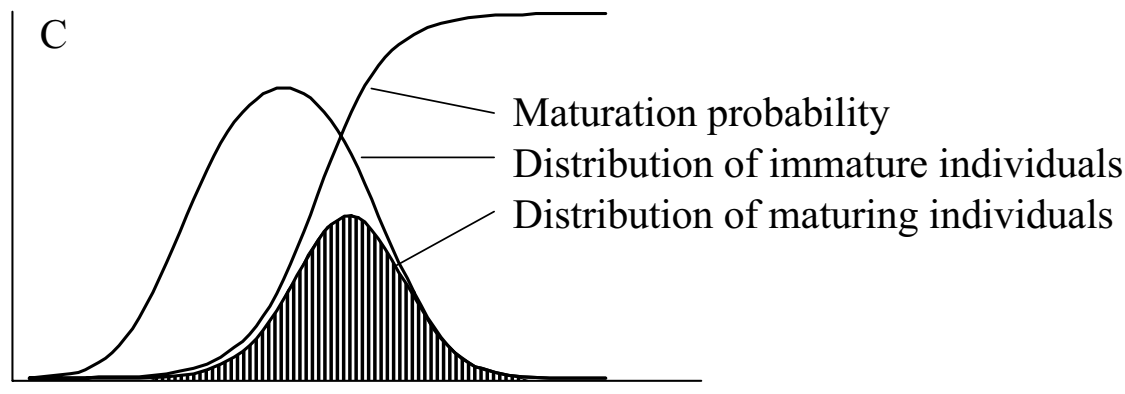

Size

Figure 1. (A) In a deterministic world, maturation would occur when a growth trajectory intersects with the reaction norm for age and size at maturation. (B) In reality, the maturation process is affected by stochastic factors, and observed combinations of age and size at maturation are therefore scattered. This variation is captured by the probabilistic reaction norm for age and size at maturation, defined as the ageand size-specific probability of maturing during a certain time interval (i.e., conditional to being immature at its beginning). The probabilistic reaction norm is illustrated by contour lines for different maturation probabilities (dots on lines). We emphasize that, as introduced in this paper, the reaction norm is defined only at discrete time intervals; the connecting contour lines are for illustration only. (C) At each age, the size-specific maturation probability translates the size distribution before maturation into the size distributions of maturing and immature individuals after maturation.

Even after observations have been made, it is still possible to estimate maturation probabilities corresponding to other time intervals, but some inaccuracy is incurred if shorter time intervals are chosen in hindsight.

The probability of maturing is generally expected to increase with size at a given age. Furthermore, this dependence will often have a sigmoid shape, such that the probability of maturing is almost zero when the size is very small, while it approaches unity when the size is very large (Fig. 1C). After selecting some suitable function for representing the shape of a probabilistic reaction norm at a given age, for example the logistic function (Fig 2), the probability of maturing can be estimated from data on proportions 


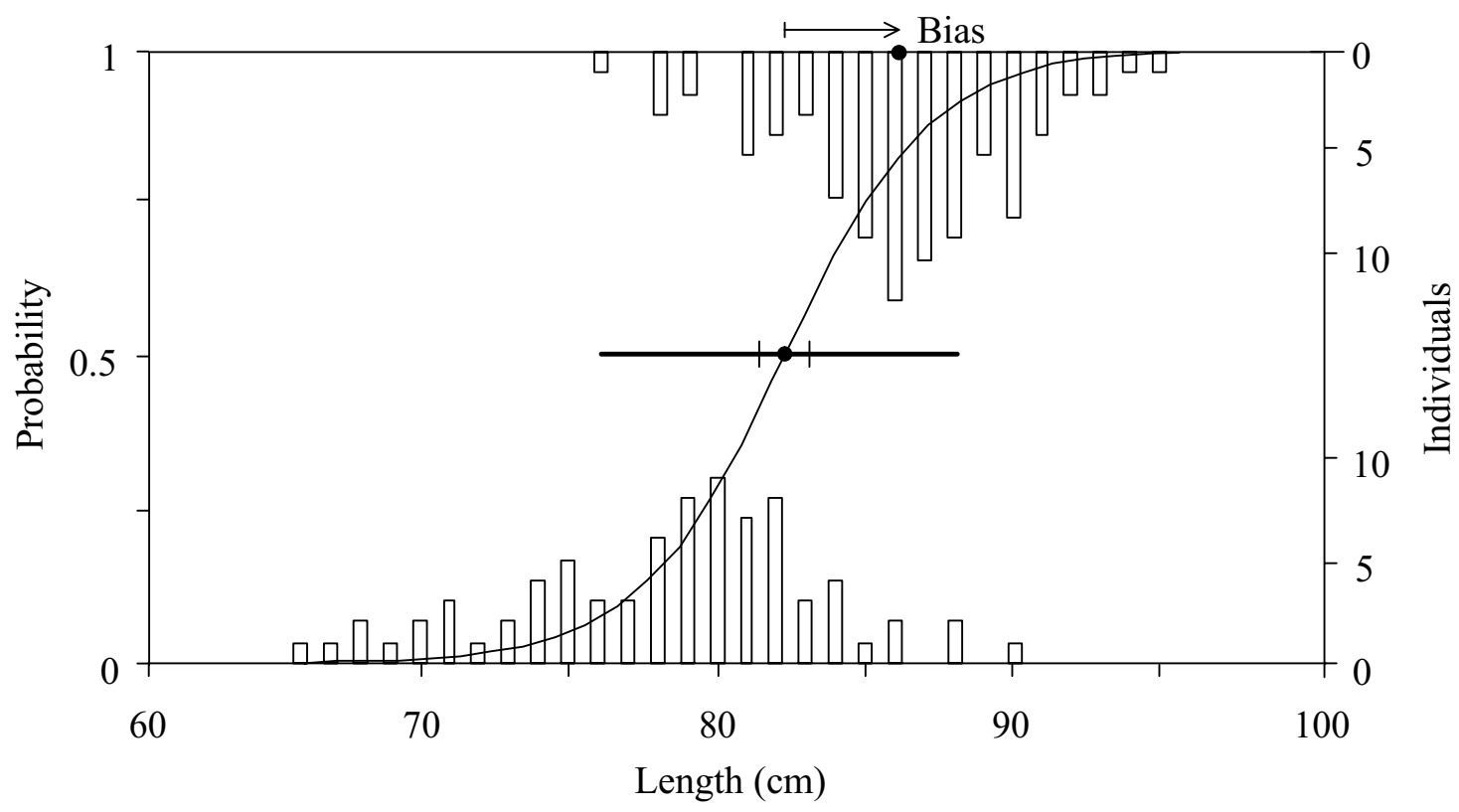

Figure 2. An example of hypothetical data on observations of maturation status (immature/maturing) and size of individuals sampled from one age group, together with the fitted logistic curve. The histogram on the top gives the size distribution of maturing individuals in the sample, and the histogram on the bottom gives the size distribution of the immature individuals. The size at which the probability of maturing reaches $50 \%$ is highlighted by the black dot on the logistic curve. The $95 \%$ confidence interval of this size is given by the horizontal error bar, and the size interval within which the maturation probability increases from $25 \%$ to $75 \%$ is shown as a thick line. The average size of maturing individuals - marked by the black dot on the top horizontal axis - is much larger than the size at which the maturation probability reaches $50 \%$. The arrow at the top draws attention to the resulting difference.

of immature and maturing individuals at a given age and size.

The full description of the probabilistic reaction norm for age and size at maturation consists of an array of functions that characterize the size-dependent maturation probabilities at all relevant ages. For descriptive purposes simpler illustrations of this array are often useful. A convenient option is to illustrate the shape of the reaction norm by contour lines that connect sizes with identical maturation probabilities at different ages (Fig. 1B). In particular, it may be convenient to capture the shape of a probabilistic reaction norm by plotting its midpoint, defined as the size at which the probability of maturing is $50 \%$, together with the lower and upper quartiles (the sizes at which the probability of maturing is $25 \%$ and $75 \%$, respectively). The length of this inter-quartile range at a given age can be used as a measure of the width of the probabilistic reaction norm. Our probabilistic approach can be considered as an extension of the classical approach: with the width of the probabilistic reaction norm decreasing, all its contour lines converge to the deterministic reaction norm as a special case.

While illustrating reaction norms graphically by contour lines, two points deserve attention. First, the chosen suite of contours should reflect the maturation probabilities observed in the data. Especially if observations are made at short time intervals, a maturation probability as high as $25 \%$ or $50 \%$ may never be observed. Second, even if contours are drawn as continuous curves for the clarity of illustration, it must be kept in mind that the probabilistic reaction norm as introduced here is defined at discrete time intervals. 


\section{Estimation of Traditional Reaction Norms}

Traditionally, there have been two major ways of estimating the reaction norm for age and size at maturation. These methods differ in whether or not observations are grouped according to growth rate.

(1) In a first approach no grouping according to growth rate is considered. This method is typically employed in analyses of data that originate from a single population. One simply plots size against age at maturation across all observations of maturation events (e.g., Policansky 1982; Stearns and Koella 1986; Chambers and Leggett 1987). The region encompassing the observed maturation events is often referred to as the maturation envelope.

(2) A second approach is suitable when observed maturation events are grouped according to growth rate. Three situations are typical. (a) An experimenter may be able to control the growth rates of studied organisms over a relatively wide range of values. A single experimental treatment would then conclude with measurements of age and size at maturation in a population, all members of which experience similar growth rates. (b) A comparable situation might arise if genetically similar but spatially separate field populations experience different average growth rates with low within-population variation. (c) Even for spatially unstructured field populations, observations could be divided into classes that correspond to different growth rate intervals. In either of these situations, a relation between age and size at maturation can be constructed by first determining the average size and age at maturation within each growth class and then plotting the resulting combinations across all classes (e.g., Gebhardt and Stearns 1988, 1993; Nylin et al. 1996; Tammaru 1998).

Both methods are very intuitive and relatively easy to apply. Notice also that both methods require data exclusively on maturing individuals. The resulting relations between age and size - possibly smoothed by fitting some regression model to the observed data points - are interpreted as representing the (deterministic) maturation reaction norm of the studied organism. Closer scrutiny, however, reveals that estimating reaction norms generally is a much more delicate task than these two traditional approaches suggest. This is because the maturation process is intrinsically stochastic and actual maturation events, at each age, will not be distributed similarly relative to the reaction norm. Instead, the population will 'sample' the maturation reaction norm in a biased way (Fig. 3). There are two independent reasons for this bias:

(1) When growth trajectories approach the reaction norm from 'below', as is the case for most organisms, maturation at the youngest age at maturation will only occur at small sizes. This is because no growth trajectories in the cohort would be steep enough to highlight the upper parts of the reaction norm at that age (Figs. 3B and 3C). Similarly, maturation events for the oldest ages at maturation will mainly occur in the upper size range if shallower growth trajectories are not equally frequent in the cohort (Figs. 3B and 3C). As a consequence of this systematic sampling bias, the observed relationship between size and age at maturation will differ from the reaction norm by exhibiting a slope that is biased towards the slope of average growth trajectory. 

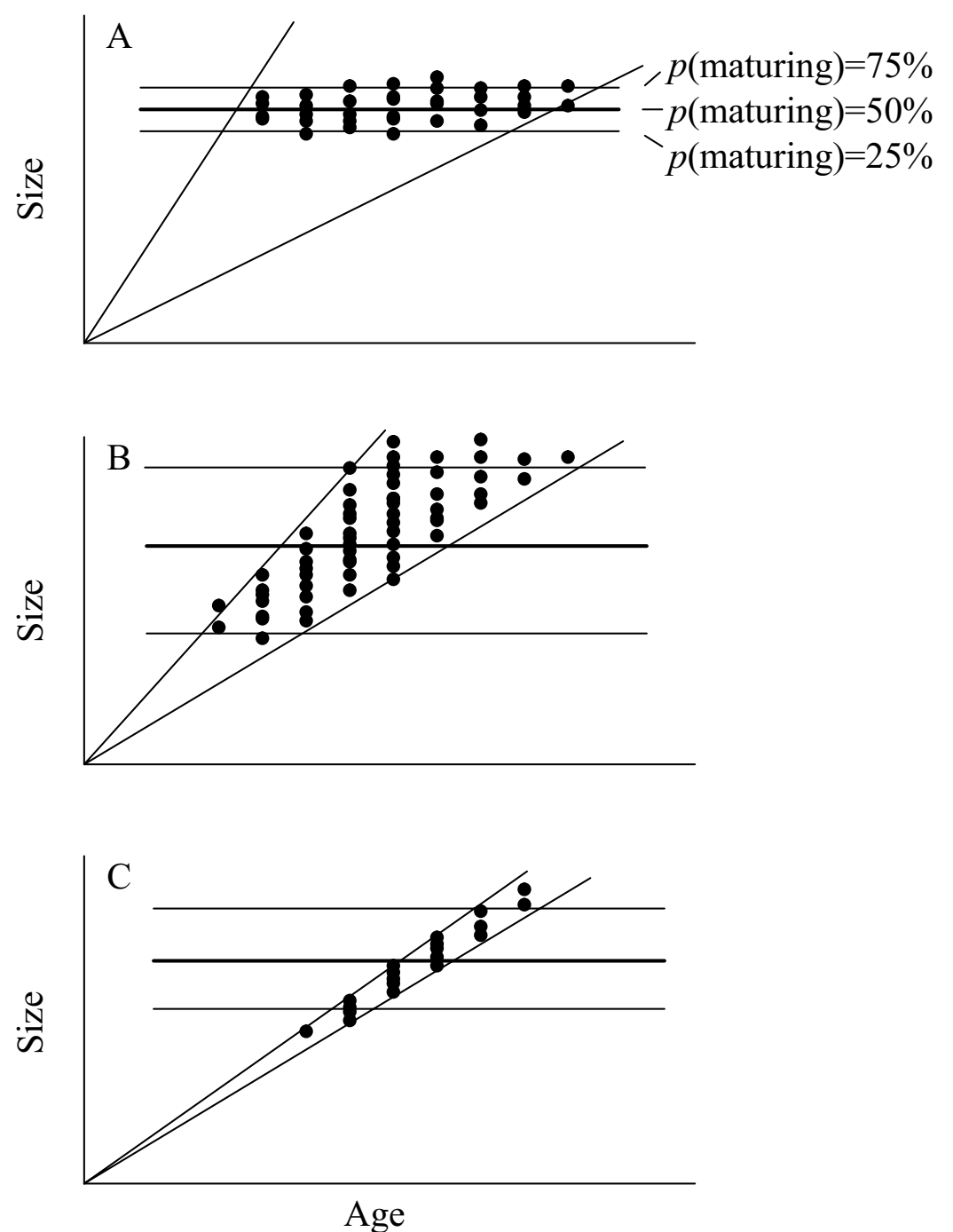

Figure 3. The interplay between the width of probabilistic maturation reaction norms (illustrated by horizontal contour lines) and the variability in the slope of growth trajectories (described by lines intersecting at the origin) in determining observed ages and sizes at maturation. (A) If the probabilistic reaction norm is narrow and variability in growth trajectories is high, plotting size against age at maturation allows for a good approximation of the probabilistic reaction norm. (B) However, if the probabilistic reaction norm is wide and growth is less variable, the observed distribution of ages and sizes at maturation becomes misleading if interpreted as a reaction norm: although the probabilistic reaction norm is horizontal, the distribution wrongly suggests a positive slope. (C) Worse, if there is hardly any variability in growth, the distribution of maturing individuals only highlights the shape of the average growth trajectory.

(2) An additional bias results from the mortality that inevitably occurs while growth trajectories pass through the reaction norm. With increasing mortality, the distribution of observed maturation events shifts downwards along the average growth trajectory. Even when mortality rates are independent of growth rates, this effect is bound to influence the observed relation between size and age at maturation. 
The narrower the distribution of growth rates is relative to the width of the probabilistic maturation reaction norm, the larger are these biases. Only when the probabilistic reaction norm is very narrow and there is large variation in growth rates, the relation between size and age at maturation provides a good approximation of the real reaction norm (Fig. 3A). At the other extreme, if the probabilistic reaction norm is wide and there is little variation in growth trajectories, plotting size against age at maturation merely highlights the growth trajectories before maturation (Fig. 3C).

We therefore conclude that only under special conditions data on size and age at maturation are sufficient for estimating reaction norms for age and size at maturation, if these are desired to be independent of growth and survival. Such special conditions might be met in experimental studies where variation in growth rates can be artificially amplified. But even then, probabilistic reaction norms of non-negligible width will systematically bias the estimates. By and large, analyzes based on plotting size at maturation versus age at maturation will give results that are misleading.

\section{Estimation of Probabilistic Reaction Norms}

Maturation is a binary response variable: individuals either mature or stay immature. The standard method for analyzing such data is logistic regression, a technique belonging to the family of generalized linear models (McCullagh and Nelder 1983; Collett 1991; Crawley 1993). Logistic regression amounts to fitting a sigmoid curve to the data (Fig. 2). In practice, logistic curves well describe the shape of probabilistic maturation curves (even though such an estimate only amounts to a phenomenological description and there is no biological reason to choose this specific function). Other choices - allowing, for example, for asymmetric shapes - are discussed in the Appendix.

The logistic curve is given by the equation

$$
\operatorname{logit}(p(s))=\log _{\mathrm{e}}[p(s) /(1-p(s))]=c_{0}+c_{1} s,
$$

where $\operatorname{logit}(p)=\log _{\mathrm{e}}[p /(1--p)]$ is the so-called logit link function, $p(s)$ is the probability of maturing at size $s$, and $c_{0}$ and $c_{1}$ are the two regression parameters of the model. In the simplest case, used in this paper for illustration, age classes are analyzed separately: in each such class, size $s$ is then the only explanatory variable (see the Appendix for examples of statistical models that incorporate several age classes). For the model above, the parameters $c_{0}$ and $c_{1}$ for each age class can be determined from data by using the method of maximum-likelihood parameter estimation. For this purpose, the data must obviously contain measurements of both maturing and immature individuals.

Denoting the estimated regression parameters for a given age class by $\hat{c}_{0}$ and $\hat{c}_{1}$, the estimated size at which the probability of maturing within the considered time interval reaches $50 \%$, is given by $-\hat{c}_{0} / \hat{c}_{1}$. Confidence intervals for these sizes can be calculated from the variance-covariance matrix of the parameter estimates, see Collett (1991). The parameter $c_{0}$ is a position parameter that shifts the fitted curve to the left or right, while the parameter $c_{1}$ describes how fast, within the considered age class, the probability of maturing increases with size; this determines the width of the maturation reaction norm. 
Other contour lines of the reaction norm, for example for $p=25 \%$ and $p=75 \%$, pass through the sizes $\left(\log _{\mathrm{e}}[p /(1-p)]-\hat{c}_{0}\right) / \hat{c}_{1}$ in each age class.

Figure 2 illustrates the use of logistic regression for estimating the probabilistic maturation reaction norm for a given age class. The original data are plotted in the form of two histograms, with individuals that remained immature at the bottom and with individuals maturing within the time interval at the top. Maturing individuals tend to be larger than those that remained immature; in particular, all small individuals are immature and all large individuals are maturing. This tendency is captured by the fitted logistic curve, which, for this age class, gives the probability of maturing during within the time interval as a function of size. The figure also shows the midpoint of the probabilistic reaction norm, and a confidence interval for the latter. Notice the conceptual difference between the width of the probabilistic reaction norm and the midpoint's confidence interval. The steeper the logistic regression curve, the narrower the probabilistic reaction norm is, and the stronger is the effect of size on maturation in the size region around the midpoint. By contrast, the smaller the confidence interval, all the more accurately the midpoint itself can be estimated from the available data.

Figure 2 illustrates the conceptual difference between the midpoint of the logistic regression curve and the mean size of maturing individuals. Growth trajectories that pass through this age class 'sample' the size range of the probabilistic reaction norm non-representatively; the mean size of maturing individuals is the result of this sampling. If the mean size at maturation is used as an estimate of the midpoint (or of some other contour of the probabilistic reaction norm), a bias arises that can be either positive or negative.

By definition, estimation of the reaction norm for age and size at maturation involves age as an explanatory variable, in addition to size. Also other explanatory variables. such as cohort, spatial location, or experimental treatment - could be included. Depending on the shape of the reaction norm, these variables can be incorporated into the statistical model in different ways. Some of these models, together with their biological interpretations, are summarized in the Appendix.

\section{Comparison of Approaches}

To illustrate the differences between the approach described here and previously used methods, we simulated maturation data for a known probabilistic reaction norm. We assumed that growth before maturation is linear and that the size of cohorts is large (we chose $N_{1}=10,000$ at age 1). Data points were generated by letting individuals grow deterministically and mature probabilistically; maturation occurred while their growth trajectories passed through the probabilistic reaction norm.

The generation procedure is based on two initialization steps and a subsequent cycle of four further steps:

1. We start from considering a probabilistic reaction norm with age-dependent coefficients $c_{0}(a)$ and $c_{1}(a)$ (eqn. 1). We select the coefficients such that they describe a linear reaction norm of fixed width; this shape is chosen to render conspicuous any discrepancies between the estimated reaction norms and the actual one.

2. The size distribution of immature individuals, $n_{\mathrm{I}}(s, a)$, at the first age considered for maturation, $a=1$, is generated for a cohort of initially $N_{1}$ individuals by picking sizes 
at random from a normal distribution with mean length of 100 units and a standard deviation of 20 units. The size distributions of maturing $\left(n_{\mathrm{I} \rightarrow \mathrm{M}}(s, a)\right)$ and mature individuals $\left(n_{\mathrm{M}}(s, a)\right)$ at all ages are initially empty.

3. Each immature individual is transferred from the immature distribution $n_{\mathrm{I}}(s, a)$ to the maturing distribution $n_{\mathrm{I}} \rightarrow \mathrm{M}(s, a)$ with probability $p$ (eqn. 1), and remains in $n_{\mathrm{I}}(s, a)$ with probability $1-p$. The resulting distributions $n_{\mathrm{I}}(s, a)$ and $n_{\mathrm{I}} \rightarrow \mathrm{M}(s, a)$ are the output of the generation procedure.

4. All individuals in the size distribution $n_{\mathrm{I}} \rightarrow \mathrm{M}(s, a)$ are transferred to the size distribution of mature individuals, $n_{\mathrm{M}}(s, a)$.

5. Immature and mature individuals survive with probability $\sigma$; the distributions $n_{\mathrm{I}}(s, a)$ and $n_{\mathrm{M}}(s, a)$ are thus multiplied with this factor.

6. If there are still immature individuals left in the cohort, we let them grow according to linear growth trajectories that pass through the origin and through the point given by the current size and age of the considered individual. We copy the resulting distributions $n_{\mathrm{I}}(s, a)$ and $n_{\mathrm{M}}(s, a)$ to the next age and return to Step 3 until no immature individuals remain in the cohort.

This procedure gives us, for a given reaction norm, the size distributions $n_{\mathrm{I}}(s, a)$ and $n_{\mathrm{I} \rightarrow \mathrm{M}}(s, a)$ of immature and maturing individuals, respectively, at all relevant ages. The probabilistic reaction norm is then estimated for each age separately with logistic regression, using all available observations.

Figures $4 \mathrm{~A}$ to $4 \mathrm{C}$ show again that the relationship between mean age and size at maturation generally bears little resemblance to the probabilistic maturation reaction norm. At early ages, a few fast-growing individuals mature at sizes where maturation probability is very low (well below the lower quartile of the reaction norm), whereas at later ages the mean size of maturing individuals is relatively high (above the upper quartile). By contrast, our technique accurately recovers the reaction norm used to generate the data, except for very early and late ages at which few individuals matured.

In general, the average age and size at maturation within a cohort does not coincide with any particular contour line of the probabilistic maturation reaction norm (Fig. 4D). If mortality before maturation is low and the probabilistic reaction norm is narrow, the combination of mean age and size at maturation may lie above the upper quartile of the probabilistic reaction norm. By contrast, if mortality is high and the probabilistic reaction norm is broad, the mean point comes to lie much below the lower quartile. Trying to estimate points on the probabilistic reaction norm by pooling observations on age and size at maturation therefore introduces yet another bias of unknown degree.

\section{Example: Reaction Norms of Northeast Arctic Cod}

We now estimate the probabilistic reaction norms for age and size at maturation from data on the Northeast Arctic cod using the logistic regression technique described above. Data has been provided by the Institute of Marine Research (Norway), based on annual routine sampling from scientific surveys and commercial catches conducted from 1985 onwards. We analyze the cohorts of 1981 to 1990, which reached maturity in the years 1985 to 1997 . This data comprises 28,387 immature and 35,614 first-time spawning fish of ages four to eight, and is the best available representation of the status of the stock in terms of size and maturity status at different ages. 

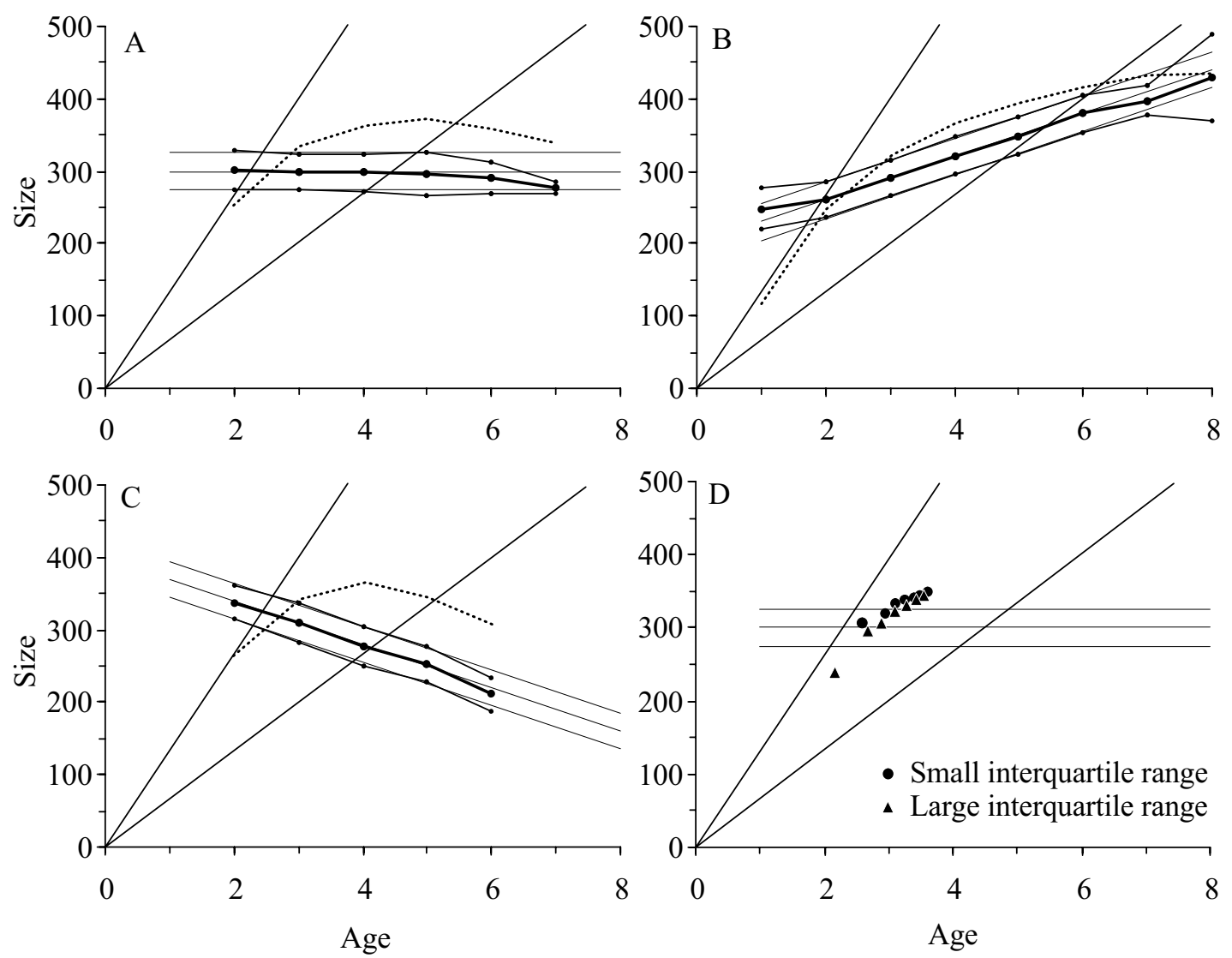

Figure 4. (A - C) Comparison of an actual probabilistic reaction norm (thin lines), estimated probabilistic reaction norms (thick lines), and mean age and size at maturation (dashed lines). Probabilistic reaction norms are illustrated with midpoint and quartiles and bear little resemblance to the mean age and size at maturation. The sector containing $90 \%$ of individual growth trajectories is shown by the two rays emerging from the origin. All three examples are based on the maturation model described in the text with an annual survival probability of $\sigma=0.8$. Ages at which less than ten individuals matured are omitted. (D) Dependence of mean age and size at maturation on mortality before maturation and on the width of the reaction norm (narrow: circles, wide: triangles). Each point shows the mean age and size at maturation for a given mortality level. Annual mortality increases from $1-\sigma=0$ to $1-\sigma=0.9$ from right to left. The higher the mortality, the lower are the mean age and size at maturation. The size interval over which the probability of maturing increases from $25 \%$ to $75 \%$ was set to 50 units for the narrow probabilistic reaction norm (shown) and to 75 units for a wide one (not shown). Probabilistic reaction norm and growth cone are depicted as in panels $\mathrm{A}-\mathrm{C}$.

To achieve a representative coverage of immature and newly matured fish, data from two different surveys was used. The winter survey, conducted in January-April in the Barents Sea, covers the immature part of the stock, as well as some mature fish that are on their way to the spawning grounds. The spring survey, carried out in March-April around the Lofoten Islands, covers the mature part of the stock. Immatures, newly matured first-time spawners, and repeat spawners are identified and aged on the basis of otolith patterns and maturation status of the gonads (Rollefsen 1933). Because of unequal sampling intensity between surveys, measured proportions of mature and immature fish typically do not reflect the actual proportions in the population as the whole. The correct proportions have been estimated by Ajiad and Jakobsen (2001) and are available as maturity ogives, giving the proportions of mature individuals for a given age and year. To conform with these ogives, over-represented types (usually the mature 
individuals) were sub-sampled randomly; results for different replicates were similar. The results below are averages across five replicates.

The probabilistic reaction norm estimated for the cohort of 1982 is shown in Figure 5A. It turns out to be dome-shaped with a roughly constant width. In contrast, the relationship between mean age and mean size at maturation is roughly linear and possesses a positive slope throughout. It is only for later ages that the mean size of newly matured fish happens to fall within the quartiles of the estimated reaction norm; otherwise there is not much resemblance.

Despite considerable variability, probabilistic reaction norms for the cohorts of 1981 to 1990 are all fairly similar and lie within the same dome-shaped band (Fig. 5B). This variability can be caused both by environmental fluctuations that directly influence maturation and by unrepresentative sampling. For some years and ages estimations do not give meaningful values; this happens primarily when samples contain either very few immature or very few newly matured individuals.

The concave shape of the established reaction norm (Fig. 5) provides important biological insight about how variation in growth influences the transition of individual fish to maturity. Fish with intermediate growth rate delay their maturation and attain similar or larger sizes at maturation than their fast-growing conspecifics. However, fish with poor growth turn out not to delay their maturation to an extent that would compensate for their slow growth. Proper understanding of plasticity in the maturation of Northeast Arctic cod may therefore provide us with precious insight into the lifehistory evolution and dynamics of this stock (Bergstad et al. 1987).

\section{Discussion}

We have in this paper introduced a new concept of the reaction norm for age and size at maturation that adequately accounts for the probabilistic nature of maturation. Estimation of these probabilistic reaction norms requires data on both immature and maturing or newly matured individuals. By contrast, the two main traditional approaches, both using the relationship between age and size at maturation, tend to result in estimations that, in most circumstances, cannot be interpreted as maturation reaction norms but instead also depend on growth and survival in the considered population. Highlighting these discrepancies, we have estimated probabilistic maturation reaction norms for the Northeast Arctic cod. The reaction norms published here are the first estimates free from the biases incurred by the traditional approaches.

A reaction norm, in general, describes how a single genotype is translated into different phenotypes in dependence on environmental conditions. In particular, the reaction norm for age and size at maturation characterizes how these two traits respond to environmental variations in growth. Notice, however, that independent environmental variables are not considered for this reaction norm. Instead, the growth trajectory followed by an individual integrates all environmental factors that affect growth into a single object, size-at-age. At each point of such a growth trajectory (which can be reached by different combinations of environmental conditions in the individual's past) the reaction norm then determines the probability of individuals at this age class and size to reach maturity during the considered time interval.

Since environmental variables are not made explicit in this description, one can argue that the maturation reaction norm should not be considered a reaction norm in the strict sense. Yet, the notion of a reaction norm for age and size at maturation has become 

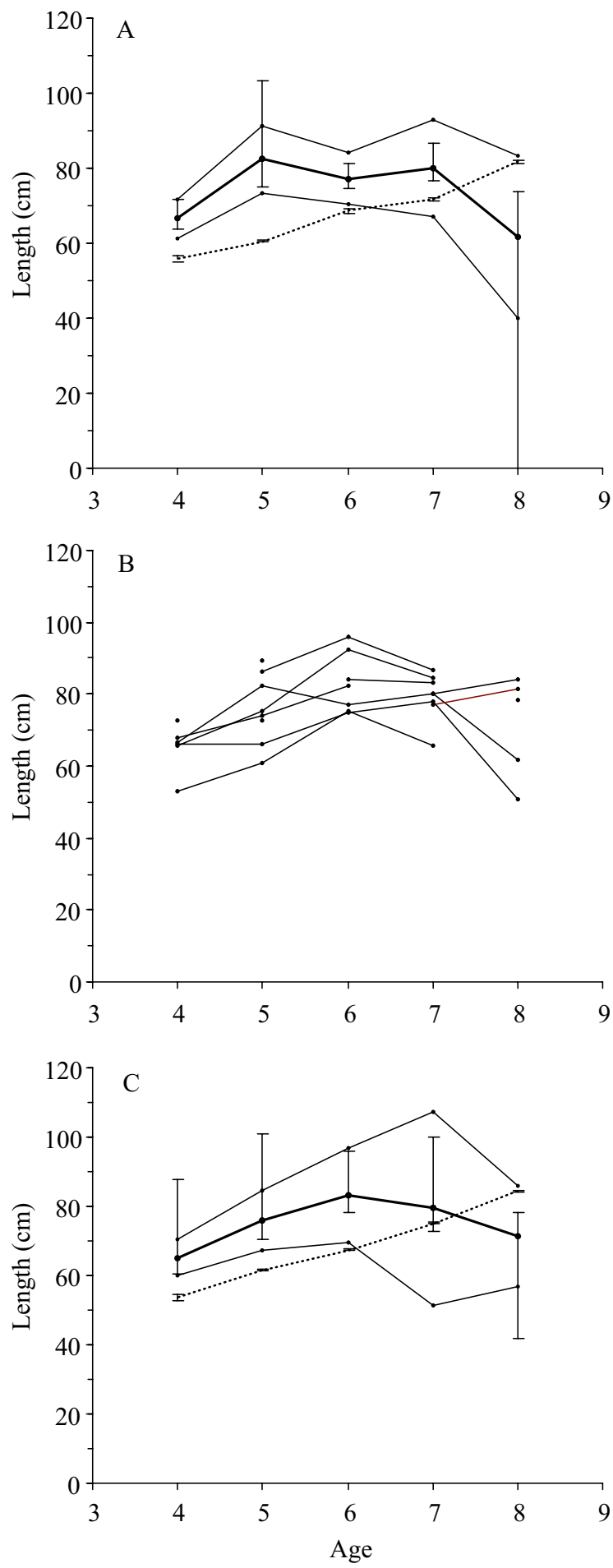

Figure 5. Probabilistic reaction norms for age and size at maturation for the Northeast Arctic cod. (A) The reaction norm for the cohort of 1981 . The thick curve shows the reaction norm midpoints (with $95 \%$ confidence intervals), and the thin curves show the quartiles. The dashed line (with $95 \%$ confidence intervals) illustrates how the mean size at maturation varies with age at maturation; like in Fig. 4, this relationship bears no resemblance to the probabilistic reaction norm. (B) Estimated reaction norm midpoints for the Northeast Arctic cod cohorts of 1981 - 1990. (C) Average probabilistic reaction norm and mean size at maturation for these cohorts. Note that the probabilistic reaction norm is defined at discrete ages; the connecting contour lines are for illustration only. 
widespread (e.g., Stearns and Koella 1986; Roff 1992; Stearns 1992; Kawecki and Stearns 1993) and we therefore decided to adhere to that convention. Nevertheless, this terminology is warranted only if the variability in growth and maturation is mainly due to environmental differences. In particular, if much variability can be ascribed to genetic variability in growth or maturation dynamics, then the maturation reaction norm may rather be a reflection of this genetic variance instead of characterizing phenotypic plasticity. This provides us with a further caveat: since reaction norms are usually envisaged to apply at the level of individuals, genetic variance in the population has to be sufficiently small as compared to environmental variance for the reaction norm to be deducible from population-level data.

Despite these caveats, the reaction norm concept has been the standard framework for understanding the relationship between age and size at maturation (Stearns and Crandall 1984; Stearns and Koella 1986). Unfortunately, differences between the actual reaction norm and the directly observed relationship between age and size at maturation have been overlooked; one reason appears to be that the importance of the probabilistic nature of the maturation process in real populations has been underestimated. Treating maturation as a deterministic process may be justified in theoretical studies (e.g., Stearns and Crandall 1984; Stearns and Koella 1986; Rowe and Ludwig 1991; Kawecki and Stearns 1993), but may set the interpretation of real data on the wrong track. As we have demonstrated above, the relationship between the mean size of maturing individuals and their age often bears little resemblance to the actual reaction norm (Figs. 3, 4, 5). The former relation may even suggest a spurious dependence of maturation on age, while the real reaction norm might be flat and maturation is ageindependent (Figs. 3C, 4A). We must therefore conclude that published maturation reaction norms are likely to be biased. That bias is directed towards the average growth trajectory; its direction and strength depends on the relative slopes of reaction norms and growth trajectories.

Published relationships between age and size at maturation (or metamorphosis) reveal an interesting pattern. Studies which rely on natural variability of growth rates tend to show relationships between age and size at maturation with slopes ranging from positive to zero and slightly negative slopes (Alm 1959; Policansky 1982; Chambers and Leggett 1987; Forseth et al. 1995; Olive et al. 1997). The steepest positive slopes thus observed are more or less equal to the slopes of the corresponding growth trajectories (a few experiments by Alm 1959; Olive et al. 1997). As we have shown above, positive slopes of the relationship between age and size at maturation that are shallower than the slope of growth trajectories unambiguously demonstrate that the actual reaction norm has at least a shallower positive slope; it may even possess a negative slope. Likewise, flat or negatively sloped relationships between age and size at maturation are clear-cut indicators of actual reaction norms with even steeper negative slopes. Thus, despite the dominance of positively sloped or flat relationships between age and size at maturation in those studies, there is no reason to believe that the corresponding actual reaction norms would follow the same pattern.

It is therefore illuminating that experimental studies in which the variability of growth rates was amplified by experimental manipulations (typically by changes in food availability or temperature) do not show a clear dominance of negative or positive relationships between age and size at maturation; indeed, many of them are non-linear (e.g., Stearns and Koella 1986; Gebhardt and Stearns 1988, 1993; Reznick 1990; Blouin 1992; Kuwamura et al. 1996; Nylin et al. 1996; Sibly et al. 1997; Blanckenhorn 1998; 
Tammaru 1998). A possible interpretation is that amplifying the variability of growth rates effectively removes much of the systematic bias and reveals a more accurate diversity of shapes and slopes of reaction norms (Fig. 3A). Although this is probably partially true, these studies raise another important problem. Typically, these studies have derived the reaction norm as the relationship between combinations of mean age and size at maturation, obtained in experimental treatments that allowed for different growth rates. Unfortunately, the combination of mean age and size at maturation may lie above as well as below the reaction norm (Fig. 4D). Thus, trying to estimate points on the reaction norm by pooling observations on age and size at maturation introduces yet another bias of unknown degree.

The probabilistic reaction norm for age and size at maturation is a more complex object to estimate (i.e., has more parameters) than the relationship between mean age and size at maturation. The confidence intervals for reaction norm midpoints can therefore be much wider than those for the mean size of maturing individuals (Fig. 5). Unbiased estimation of the probabilistic reaction norm sets high requirements on the utilized data: First, it is necessary to know the size distributions of immature and maturing individuals. Second, the proportions of immature and maturing individuals in the samples should be representative. At first sight, these requirements seem to preclude the estimation in many interesting systems. However, alleviating the requirements is possible and often practical. First, if size distributions of immatures are unavailable, these can be reconstructed from proportions of mature individuals at age and from knowledge about immature growth patterns (M. Heino, U. Dieckmann, O. R. Godø, in prep.). Second, if individuals that have just reached maturity cannot be distinguished from those that matured earlier, it is possible to use an alternative estimation method that is based on data on immature growth and on estimated proportions of mature individuals at age and size (S. Barot, M. Heino, L. O'Brien and U. Dieckmann, in prep.).

The principles of estimating reaction norms for age and size at ontogenetic transitions other than maturation are the same as those outlined in this paper - practical problems, however, may differ. For example, when the transition involves metamorphosis, morphological changes may make it difficult to compare the sizes of individuals before and after metamorphosis. A first option then is to observe individuals continually and to measure their sizes upon entering metamorphosis. Alternatively, one can control for the mortality occurring between censuses. A second option is to measure individuals after completing their metamorphosis. In the first case, the estimation technique presented in this paper can be applied directly. In the second case, a mapping between sizes just before and just after metamorphosis must be established empirically; based on that, our method is again applicable.

Using simple logistic regression for estimating the reaction norm for age and size at maturation is adequate for data to which each measured individual has contributed only once. This case is typical when samples are taken from large populations and/or when sampling is destructive. However, if it is practical to follow individual trajectories of growth and development, a single individual can contribute several observations; the resultant consecutive observations are then correlated. The models presented here do not account for such complexity and statistical methods that accommodate repeated measures (e.g., Diggle et al. 1994) must be used to refine them. Sampling with repeated measurements, when practical, would actually appear to be the most powerful approach to estimating maturation reaction norms. Comparing the relative merits of different 
experimental designs is an attractive challenge for future research, but unfortunately lies beyond the scope of this paper.

One might be tempted to think that, since the relationship between age and size at maturation has been the standard for measuring the maturation reaction norm, there is no good reason to adopt the new probabilistic definition. The relationship between age and size at maturation even has the benefit of demanding less data. However, there are several drawbacks of that definition.

First, no mechanistic interpretation can be given to a relationship between age and size at maturation - in other words, this relation cannot be directly linked to the individual-level maturation process. Second, the relationship between age and size at maturation jointly depends on processes of maturation, growth, and survival. Resulting from a mixture of effects at the level of the population and the environment, such relationships cannot be used to distinguish between properties of populations (and thus, ultimately, of individuals) and properties of their environments. Even if all individuallevel and population-level properties remain unchanged, the relationship between age and size at maturation varies with environmentally induced modifications in average growth rates or mortality rates, or even in the variation around them (Fig. 3). Third, the relationship between age and size at maturation is not suitable as an input to age- and size-structured population models, which, instead, require knowledge about the probabilistic reaction norm. The need to use such models arises, for example, in the context of fisheries assessment and management where more realistic treatment of growth and maturation dynamics are needed to improve predictions of recruitment and sustainable yield.

Despite its obvious shortcomings, the relationship between age and size at maturation will remain a useful descriptive statistics. In the absence of proper data on immature and maturing individuals one may even not be able to penetrate further. Even then, however, interpretation of relationships between age and size at maturation is facilitated by awareness of the fact that these relationships depend both on the probabilistic reaction norm and on how a population 'samples' this reaction norm.

In summary, the probabilistic reaction norm for age and size at maturation is needed whenever one intends to describe the biological process of maturation independently of the processes of growth and mortality. A sound understanding of maturation dynamics in natural populations will therefore require accurate and unbiased estimations of probabilistic maturation reaction norms. With these reaction norms playing a central role for the dynamics of populations in temporally or spatially heterogeneous environments, achieving such understanding is an important step toward successfully managing and conserving populations in the wild. 


\section{Appendix: Logistic-Regression Models for Maturation Reaction Norm}

There are many different ways to incorporate age and size as explanatory variables into statistical models devised to estimate probabilistic reaction norms for age and size at maturation. These models differ in their level of complexity, both biological and statistical. Below we present the most important models in the order of increasing complexity.

In the first four models, age is treated as a continuous variable. The simplest statistical model involving both age and size assumes that these two variables have independent, linear effects on the probability $\mathrm{p}$ of an individual at age a and with size $\mathrm{s}$ to mature:

$$
\operatorname{logit}(p)=c_{0}+c_{1} s+c_{2} a,
$$

where the $c$ 's are the parameters of the model that are estimated from the data and the link function logit is given by $\operatorname{logit}(p)=\log _{\mathrm{e}}[p /(1-p)]$. In this model, the contours of the probabilistic maturation reaction norm are linear and its width is independent of age. The width of the probabilistic reaction norm is allowed to become age-dependent by including an interaction term between age and size:

$$
\operatorname{logit}(p)=c_{0}+c_{1} s+c_{2} a+c_{3} s a .
$$

Note that in this model both the reaction norm midpoint and the width are monotonically varying with age. Any non-monotonic dependence of the probabilistic reaction norm's contour lines on age requires the introduction of a higher-order age term. The simplest such models is:

$$
\operatorname{logit}(p)=c_{0}+c_{1} s+c_{2} a+c_{3} a^{2} .
$$

This model allows for humped or dome-shaped probabilistic reaction norms, but assumes that the width of the reaction norm is constant with age. Age-dependence of the width can again be introduced by including an interaction with age and/or age-squared:

$$
\operatorname{logit}(p)=c_{0}+c_{1} s+c_{2} a+c_{3} a^{2}+c_{4} s a+c_{5} s a^{2} .
$$

All the above models force the contour lines of the probabilistic reaction norm either to be monotonic or to possess a single maximum or minimum. Reaction norms of more complex shape require the addition of further higher-order terms. Age can also be used as a factor (i.e., classifying variable). The simplest such model is:

$$
\operatorname{logit}(p)=c_{0}+c_{1} s+c_{2, \mathrm{a}}=c_{0, \mathrm{a}}^{\prime}+c_{1} s,
$$

where $c_{2, \mathrm{a}}$ (or $c_{0, \mathrm{a}}^{\prime}$ ) are constants estimated for each age group. This model imposes no constraints on the overall shape of the probabilistic reaction norm, but its width is constant with age. Again, flexibility of the reaction norm's width with age is achieved by adding an interaction term:

$$
\operatorname{logit}(p)=c_{0}+c_{1} s+c_{2, \mathrm{a}}+c_{3, \mathrm{a}} s=c_{0, \mathrm{a}}^{\prime}+c_{1, \mathrm{a}}^{\prime} s .
$$

This model sets no constraints whatsoever on the shape of the probabilistic reaction norm, and its contour lines can vary sharply between age classes.

All the above models assume the same basic size-dependent shape for the probabilistic reaction norm: it is symmetric around the reaction norm midpoint and 
determined by the logistic curve (Fig. 2). To make this relationship more flexible (e.g., to allow for asymmetric shapes), the explanatory variable size can be transformed, or a link function other than logit can be assumed.

When selecting the appropriate statistical model for a given data set, one will usually want to start from the simplest model and then proceed towards more complex ones. Often there are alternative paths that could be followed, and the model selection is a delicate task that should be guided by both biological and statistical considerations. For detailed accounts on model selection see McCullagh and Nelder (1983), Collett (1991), and Crawley (1993). 


\section{References}

Ajiad, A. and T. Jakobsen, 2001. Historical time series for Northeast Arctic cod. ICES Working Group Meeting/WD 1. ICES, Copenhagen.

Alm, G. 1959. Connection between maturity, size, and age in fishes. Rep. Inst. Freshw. Res. Drottningholm 40:5-145.

Bergstad, O. A., T. Jørgensen, and O. Dragesund. 1987. Life history and ecology of the gadoid resources of the Barents Sea. Fish. Res. 5:119-161.

Bernardo, J. 1993. Determinants of maturation in animals. Trends Ecol. Evol. 8:166-173.

Blanckenhorn, W. U. 1998. Adaptive phenotypic plasticity in growth, development, and body size in the yellow dung fly. Evolution 52:1394-1407.

Blouin, M. S. 1992. Comparing bivariate reaction norms among species: time and size at metamorphosis in three species of Hyla (Anura: Hylidae). Oecologia 90:288-293.

Chambers, R. C., and W. C. Leggett. 1987. Size and age at metamorphosis in marine fishes: an analysis of laboratory-reared winter flounder (Pseudopleuronectes americanus) with a review of variation in other species. Can. J. Fish. Aquat. Sci. 44:1936-1947.

Collett, D. 1991. Modelling binary data. Chapman \& Hall, London.

Crawley, M. J. 1993. GLIM for ecologists. Blackwell Scientific, Oxford.

Diggle, P. J., K.-Y. Liang and S. L. Zeger. 1994. Analysis of longitudinal data. Oxford Univ. Press, Oxford.

Forseth, T., O. Ugedal, and B. Jonsson. 1995. Inter and intra cohort variation in the life history of Arctic charr (Salvelinus alpinus). Nordic J. Freshw. Res. 71:237-244.

Gebhardt, M. D., and S. C. Stearns. 1988. Reaction norms for developmental time and weight at eclosion in Drosophila mercatorum. J. Evol. Biol. 1:335-354.

Gebhardt, M. D. 1993. Phenotypic plasticity for life history traits in Drosophila melanogaster. I. Effect of phenotypic and environmental correlations. J. Evol. Biol. 6:1-16.

Kawecki, T. J., and S. C. Stearns. 1993. The evolution of life histories in spatially heterogeneous environments: Optimal reaction norms revisited. Evol. Ecol. $7: 155-174$.

Kuwamura, T., Y. Nakashima, and Y. Yogo. 1996. Plasticity in size and age at maturity in a monogamous fish: effect of host coral size and frequency dependence. Behav. Ecol. Sociobiol. 38:365-370.

McCullagh, P., and J. A. Nelder. 1983. Generalized linear models. Chapman \& Hall, London. 
Nylin, S., K. Gotthard, and C. Wiklund. 1996. Reaction norms for age and size at maturity in Lasiommata butterflies: predictions and tests. Evolution 50:1351-1358.

Olive, P. J. W., J. Fletcher, S. Rees, and G. Desrosiers. 1997. Interactions of environmental temperature with photoperiod in determining age at maturity in a semelparous polychaete Nereis (Neanthes) virens Sars. J. Therm. Biol. 22:489-497.

Policansky, D. 1982. Influence of age, size, and temperature on metamorphosis in the starry flounder, Platichtys stellatus. Can. J. Fish. Aquat. Sci. 39:514-517.

Reznick, D. N. 1990. Plasticity in age and size at maturity in male guppies (Poecilia reticulata): an experimental evaluation of alternative models of development. J. Evol. Biol. 3:185-203.

Roff, D. A. 1992. The evolution of life histories. Theory and analysis. Chapman \& Hall, New York.

Rollefsen, G. 1933. The otoliths of the cod. FiskDir. Skr. Ser. HavUnders. 4:1-14.

Rowe, L., and D. Ludwig. 1991. Size and timing of metamorphosis in complex life cycles: time constraints and variation. Ecology 72:413-427.

Sibly, R. M., L. Winokur, and R. H. Smith. 1997. Interpopulation variation in phenotypic plasticity in the speckled wood butterfly, Pararge aegeria. Oikos 78:323-330.

Stearns, S. C. 1992. The evolution of life histories. Oxford Univ. Press, Oxford.

Stearns, S. C., and R. E. Crandall. 1984. Plasticity for age and size at sexual maturity: a life-history response to unavoidable stress. Pp. 13-33 in G. Potts and R. J. Wootton, eds. Fish reproduction. Academic Press, London.

Stearns, S. C., and J. C. Koella. 1986. The evolution of phenotypic plasticity in lifehistory traits: predictions of reaction norms for age and size at maturity. Evolution 40:893-913.

Tammaru, T. 1998. Determination of adult size in a folivorous moth: constraints at instar level? Ecol. Entomol. 23:80-89. 